\title{
See the Apple of My Eye: Baby Storytelling in Social Space
}

\author{
N. Jomhari \\ University of Malaya \\ Faculty of Computer Science \& IT \\ Kuala Lumpur, Malaysia \\ nazean@um.edu.my
}

\author{
V. M. Gonzalez \\ University of Manchester \\ Manchester Business School \\ Manchester, United Kingdom \\ vmgonz@manchester.ac.uk
}

\author{
S. H. Kurniawan \\ University of California Santa Cruz \\ Computer Engineering \\ Santa Cruz CA 95064, USA \\ srikur@soe.ucsc.edu
}

\begin{abstract}
People use media-sharing web sites to document their lives and those of their children for maintaining and strengthening social ties with people living away. It is clear then that people can and like to create narratives as a form of expression. This study presents an analysis of the characteristics and type of baby stories written by young mothers. Nine mothers from Malaysia living in the UK participated in the study. The participants used a variety of media-sharing web sites to prepare and share the narratives. Most of them (seven) used photo-sharing web sites (Fotopages or Flickr), two used text-based blogs (Blogger). Two of them also uploaded videos of their babies in content sharing sites (YouTube). Within the period of three months, we identified 166 stories created, with 94 percent of them focusing on their baby. The stories present a number of topics such as skills demonstrations, outings, domestic activities, and social events. Based on the analysis of the data and interviews with participants, we found a significant positive correlation between the type of story and the type of media used. The result also shows there was a significant positive relationship between the type of story and the baby's age.
\end{abstract}

\section{Categories and Subject Descriptors}

H.5.3 [Information Interfaces and Presentation]: Group and Organisation Interfaces - Asynchronous interaction, evaluation

\section{General Terms}

Design, Human Factors.

\section{Keywords}

Media-sharing, narratives, baby stories, mothers, ComputerMediated Communication (CMC).

\section{INTRODUCTION}

In modern society, more and more people have experienced the challenge of communicating and maintaining long distance relationships due to business, marriage, military deployment, working or studying. The development of Information and Communications Technologies in the past decades has contributed to facilitate the communication with colleagues, clients, family and friends. Our research focuses on family communication as maintenance of strong ties with friends and family are of uppermost importance for many people living

(C) The Authors 2009.

Published by the British Computer Society abroad [1]. In particular, we analyze technology usage within the context of young families wanting to share the life of their babies with extended family members living away.

Narratives of everyday experiences are said to be relevant in keeping families living apart emotionally connected. For instance, Van House's study of photo-sharing practices in Flickr.com reveals how photos enhance conversations among distant relatives, and thereby providing the means to keep them aware about daily activities [2]. Similarly, Nardi et al. found that people use blogging web sites to document their lives and those of their children as well as to maintain and strengthen social ties with people living away [3]. Corson suggests that grandparents with limited opportunities for seeing regularly their grandchildren can record videos of them telling a story as a way to enhance connectedness [4]. With new video sharing technology like YouTube.com, video recordings are much easier to deliver. Furthermore, recent studies by Gonzalez and Kurniawan [5] and Harley and Fitzpatrick [6] have shown that YouTube can be a suitable tool for intergenerational communication. Both studies examined the YouTube user Geriatric1927, an older adult who updates his blogs in YouTube. They highlighted the value of video-based communication to share experiences, avoid isolation, and facilitate dialogue.

Each of the media discussed above can facilitate different ways of baby storytelling, depending on the characteristics of the story and people's preferences. As the Media Richness Theory suggests, the choice of media is dependent upon the communication task being attempted [7]. The theory states that people choose a certain medium to perform a particular task, and that the use of a particular medium is dependent on the task. Media choice depends on the perceived effectiveness of the media to present the information for particular needs. In the context of preparing stories, it can mean that some stories might need additional information (medium) to make them clear to the reader. For example, to show that a baby can walk, video would be a suitable media.

The high or low richness of a media depends on four criteria: feedback, multiple cues, language variety, and personal focus. Written media is suitable for clear messages and unambiguous information. Text media is categorized as having low richness because it has fewer cues, less language variety (only text) and is low in personal focus of emotion. Photo-stories may help to describe textual information to give a clearer picture. The video story is the richest media as it contains multiple cues (movement, sound, visual and potentially text), high in language variety (people with other languages can understand it by looking at the video, especially when it involves movement) and high in personal focus of emotion because one can hear the baby cry or can see the movement that conveys emotions.

Based on the richness of media we can expect that digital narratives will fulfil different purposes, will have different 
characteristics and will result in different practices. We aim at investigating the making of these narratives and our departure point was the analysis of current usage of a number of mediasharing technologies.

Our study is guided by two main research questions:

1. What are the types of stories created by people wanting to share the life of a baby?

2. What are the practices characterizing this form of digital story telling?

We chose new mothers as the target participant group as mothers have been found to be a reliable source for news on baby development and more interested in maintain family media records [8]. The rest of this paper is organized in the following way: Section 2 describes the characteristics of the community investigated: Malaysian students in the UK; Section 3 discusses how digital technologies support the making of storytelling. Section 4 presents the content analysis on the participants' social network websites which reported the types of baby storytelling. Section 5 presents narrative practice towards maintaining their social networking for their baby. Finally, Section 6 presents our conclusion and discussion and future work

\section{MALAYSIAN IN THE UK}

The Malaysian Student Overseas Statistics Report 2002-2007 published by the Malaysian Ministry of Higher Education (MOHE), states that the UK and Ireland are the most favourite places to study after Australia [9]. In 2004, MOHE released a new initiative to ensure that at least $75 \%$ of the lecturers in public institutions of higher education possess a $\mathrm{PhD}$ qualification or its equivalent. As part of this initiative MOHE has offered scholarships and allowed more government staff to move abroad for a period with the purpose of obtaining certified training or academic degrees. As a result of this plan, Malaysians studying in the UK and Ireland has maintained a sustained grow since 2005. Even though the statistics shows that the number of Malaysian students will slowly decrease due to economy turn down, the total of number of students in the UK and Ireland was about 11,500 in 2007. It is reported that a majority of the Malaysian mature students are women [10]. In general, Malaysians establish their families and marry when they reach their mid-twenties. In the year 2000, a census of Malaysian population and housing showed that the mean average age of people getting married was 28.6 years for men and 25.1 years of age for women [11]. Therefore it is common that Malaysians studying abroad are with their spouses and become parents either shortly before leaving or while living in another country.

\section{DIGITAL TECHNOLOGIES SUPPORT THE MAKING OF STORYTELLING}

User-generated content is a common trend that defines the recent use of the Internet. For instance, in the USA according to PEW Internet and American Life Project in March 2008, a sizable proportion $(43 \%)$ of families with broadband Internet like and typically share and post on the Internet something that they created. This includes media such as photos, life stories, or videos [12].

Within the context of media-sharing websites, our study focuses on the use of these technologies for storytelling of babies' lives. Baby storytelling and narratives of everyday life as a form of content creation is increasingly popular in both generic and purpose specific websites and social networking spaces. Major newspapers such as the Washington Post report that more and more young parents share their baby stories in social spaces such as Facebook [13]. A host of new sites, including Totspot, Odadeo, Lil'Grams and Kidmondo, now offer parents a chance to, instead of sending multiple e-mails messages with their baby stories, invite friends and family to join and contribute to a network geared to connecting them to their babies. In addition there are also websites specifically for documenting baby lives; among them BabyJellyBeans, OurBabyHomepage or TotSites. In spite of their lack of specificity, other generic media-sharing web sites such as Fotopages, Blogger, YouTube or Flickr are also likely to be used to create baby narratives with text, photos or videos. This trend to enhance family communication with media sharing is also visible in many research projects. Although not extensively tested, a number of systems have been proposed for supporting narrative and storytelling creation with the purpose of connecting distant families with no collocated audiences e.g. $[14,15]$ It is apparent that people can and like to create narratives as a form of expression. Yet we still need to consolidate a clear understanding of the role played by different media and tools on the creation of those narratives, as well as about the nature of digital storytelling around family life.

\section{CONTENT ANALYSIS OF CURRENT SOCIAL SPACE}

Nine mothers from Malaysia living in the UK and using some type of media-sharing social space participated in the study. We conducted content analysis (CA) of the stories posted (either based on text, picture or video) by our participants in their social space for three months (December 2007 until end of February 2008). CA on people's activity on social space has been proposed by Herring (2004) for the study of people's interaction with new media because it emphasizes on "what people do, rather than what they say they do" [16].

Six of the mothers participating in our study are working and studying. Three of them are housewives (on unpaid leave from their jobs to accompany their husband studying). However most of them worked in managerial and professional level back in Malaysia; eleven of them were lecturers, two were engineers and others were accountant, doctor and administrative officer. Their range of age is from 25 to 34 years old. Most of them have just one baby, two participants have two children and only one participant with three children (but we focused on the youngest of the babies). At the time of the study, three of them have children below three years old, three with babies age one to two years old and three with newborn babies (below one year old).

We found that among the nine participants, all of them posted regularly digital content on media-sharing web sites. Five participants used Fotopages, two used Blogspot account (linked with Picasa for image storage) and two used Flickr. In addition, two of them uploaded their baby video in YouTube too. Generally the entire group of participants update their social space at least twice a month.

We analyzed the media content to identify the particular stories that were communicated. The story is counted according per entry for the text story and video story. However for photobased stories, and following methods applied in previous studies, we categorized according to the date taken, background of the picture and also the text description which can be seen in sequence of stories [17]. Stories which are not related to baby life, or have just one photo, were discarded. Sixteen stories not related with the baby were discarded. Among them, we found 
one story about a wedding anniversary, another story about a football match, an entry about a song and lyric, one entry about a commercial product, two stories about car and nine stories about recipes. Interestingly and similarly to other studies of photo logging practices, we found that our participants in general tend to post happy moments and choose the best of their pictures $[18,19]$. The total number of baby stories analyzed was 150 , which is 94 per cent of the total of our sample. The fact that the majority of people are baby-related is indicative of the relevance of this topic for these families.

Type of stories. We defined a coding scheme to categorize the baby stories based on the scheme proposed in the Digital Imaging Consumer Survey in 2000 [20]. From our data samples we defined a coding scheme with eight categories as shown in Table 1. The four top categories are Portraits \& Performances, Outings, Domestic Activities and Special Events. Portrait and Performances is where the story focuses on the baby's face and body movements (either alone or with other family members) see Figure 1. Only the photo stories fall into this category. The difference between Outings and Holidays are the distance and time spent. Outing was usually done around Manchester and involved activities such as shopping, going to the stadium, museum, or park; where they did not stay overnight. Holidays are usually activities that are further from Manchester or that require them to stay overnight. We suspect that Portrait \& Performance was the most frequent topic as the study was performed in winter and therefore the participants spent most of the time indoors with family at home. Domestic activities are in-house's activities, e.g., playing with toys, meal-time, watching television. We found a statistically highly significant difference between the distribution of story groups from the existing stories in participants' social space and that which would be expected from a random sample $\left(X^{2}=123.391, \mathrm{df}=8\right.$, $\mathrm{p}<0.0001)$.

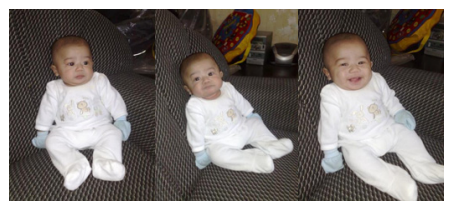

Figure 1: Portraits

After we identified the type of story, we mapped it according to the media (text, photo or video) to see relationship between the type of story and the media used (see Table 2). The result shows that there was a significant positive correlation between the type of story and the type of media used $\left(X^{2}=112, d f=14\right.$, $\mathrm{p}<0.0001)$. Majority of the participants like to post photos (59 per cent), second is text stories ( 28 per cent) and lastly videos (19 per cent). Text story is more about the Outing experience, while majority photo are about Portraits \& Performances and video story preferred for Domestic activities (see Table 2). Domestic activities also involve activities on basic skill where the baby learns to do basic physical development activity such as walking, eating or rolling.

We also investigated the relationship between the types of story with the baby's age group (see Table 3 ). The result shows that there was a significant positive correlation between the type of story and the baby's age $\left(X^{2}=79.5, d f=14, p<0.0001\right)$. Baby aged below one year old are dominantly in the Portraits \& Performance category. One reason is the baby is still too young to do physical activities or to go outing in cold weather. Outings stories appeared quite frequently in babies aged one to three. We believe that the frequent appearance of Outings stories was influenced by the long Christmas holiday in
December with majority of the participants taking this opportunity to do outings with their families.

Table 1. Type of baby stories

\begin{tabular}{|c|c|c|}
\hline Type of story & \multicolumn{2}{|c|}{ \# stories } \\
\hline Portraits \& Performances* $^{*}$ & 59 & $39 \%$ \\
\hline Outings & 30 & $20 \%$ \\
\hline Domestic activities & 19 & $13 \%$ \\
\hline Special event & 18 & $12 \%$ \\
\hline People visiting & 8 & $5 \%$ \\
\hline Birthday & 7 & $5 \%$ \\
\hline Holidays & 6 & $4 \%$ \\
\hline Nursery & 3 & $2 \%$ \\
\hline Total & $\mathbf{1 5 0}$ & $\mathbf{1 0 0} \%$ \\
\hline * Photo only & \multicolumn{2}{|}{}
\end{tabular}

Table 2. Type of baby stories and media.

\begin{tabular}{|c|r|r|r|c|}
\hline Type of story & \multicolumn{1}{|c|}{ Text } & \multicolumn{1}{c|}{ Photo } & Video & Total \\
\hline $\begin{array}{c}\text { Portraits \& } \\
\text { Performances* }\end{array}$ & 0 & 59 & 0 & 59 \\
\hline Outings & 16 & 11 & 3 & 30 \\
\hline Domestic activities & 5 & 5 & 9 & 19 \\
\hline Special event & 11 & 4 & 3 & 18 \\
\hline People visiting & 1 & 7 & 0 & 8 \\
\hline Birthday & 2 & 1 & 4 & 7 \\
\hline Holidays & 4 & 2 & 0 & 6 \\
\hline Nursery & 3 & 0 & 0 & 3 \\
\hline Total & $\mathbf{4 2}$ & $\mathbf{8 9}$ & $\mathbf{1 9}$ & $\mathbf{1 5 0}$ \\
\hline
\end{tabular}

Table 3. Type of baby story and baby's age group

\begin{tabular}{|c|c|c|c|c|}
\hline Type of story & Age $<=\mathbf{1}$ & $\mathbf{1}<=\mathbf{2}$ & $<\mathbf{3}$ & Total \\
\hline $\begin{array}{c}\text { Portraits and } \\
\text { Performances* }\end{array}$ & 59 & 0 & 0 & 59 \\
\hline Outings & 14 & 6 & 10 & 30 \\
\hline Domestic activities & 13 & 1 & 5 & 19 \\
\hline Special event & 10 & 2 & 6 & 18 \\
\hline People visiting & 7 & 0 & 1 & 8 \\
\hline Birthday & 6 & 1 & 0 & 7 \\
\hline Holidays & 0 & 4 & 2 & 6 \\
\hline Nursery & 0 & 0 & 3 & 3 \\
\hline Total & $\mathbf{1 0 9}$ & $\mathbf{1 4}$ & $\mathbf{2 7}$ & $\mathbf{1 5 0}$ \\
\hline
\end{tabular}

\section{NARRATIVE PRACTICE}

We aim at investigating the topics participants thought as worth sharing but also, as suggested by Hyvärinen (2008) to study the narrative practice too, the way that content is crafted and presented [21]. Interviews with our participants have been used to gain information on their narrative practices and family social life. To guide the analysis of our data we followed a narrative practice categorization scheme proposed by David et al.(2007) who studied users' practices with video sharing and identified a four level of lifecycle; pre-capture, at-capture, postcapture and end use [22].

1. Pre-capture. Capture is for video and the photo taking only. For written story the participants said she composed the idea in mind with the intention to transform it to words in computer later. The camera ensured to be with them at all times as not to 
miss the perfect moment to be captured. Five of them are using Digital Single Lens Reflex (DSLR) camera while the rest using compact digital camera. But the husbands are also helping with the capturing too.

"While I lulled my baby at night, the idea to write about her is already in my mind just waiting to be transferred to the computer." (Mother of 1 year old boy)

"Sometimes I didn't plan to capture his picture. Sometimes he keeps on smiling or laughing, the cute ones will quickly grab my attention." (Mother of 7 months baby)

"I'm not thinking or planning to capture any video of him, it is spontaneously happening...I just enjoy doing it." (Mother of 5 months baby and 4 years old boy)

"I like to video recording only if something special happens. For example if I tell people my son reciting pre-sleep prayer, people may not understand it if I present in text or photo, but if I show video people can understand it easily." (Mother of 3 years old boy and 5 years old girl)

2. At-capture. At capture involves the activities using the device. However based on the interview during the capture activities, the findings below are discovered:

"I always keep my digital camera with me, or sometimes when the battery is out the hand phone camera also would do" (Mother of 4 months baby and two boys)

"The video is hard to capture while we went outing so I'd prefer just taking picture" (Mother of 3 years old boy and 5 years old girl)

"We capture the video mostly at home while the baby is playing with toys or sometimes my husband capture the video and I will encourage the baby to make sound or laugh" (Mother of 5 months baby and 4 years old boy)

"The video stop recording depending on the actor (baby), if he does not want to act anymore (no mood) the director has to stop recording" (Mother of 5 months baby and 4 years old boy) 3. Post-capture. All the participants transfer the photo or video straight to the computer. However the transfers usually occur either early in the morning or late at night while the baby is sleeping. Post capture also involves the editing process in the computer.

"Besides following the folder from digital camera, I sometimes create my own folder on computer and name it according to the place we visited plus the date too." (Mother of 3 years old girl)

"I update my blog usually after the morning prayer while he (the baby) is sleeping, if not he will be jealous (disturbed) if see me with my laptop." (Mother of 1 year old boy)

"My husband will transfer the data to computer after the memory card is full. So he uploads all the pictures from born until now." (Mother of 1 year old boy)

"If I am stressed or feel bored of studying I would edit and touch up my baby photos in Photoshop. I don't do it once at a time, I just like doing all those things" (Mother of 3 years old girl).

"It depends, if we went for a holiday for five days so I will update my Fotopages five days (like day 1, day 2..) according to what activities we had done on the particular day" (Mother of 3 years old boy and 5 years old girl)

"I upload the pictures first, then it gives me idea to write about the description about the places, usually it takes about one short paragraph. I prefer the combination of these two (text and picture" (Mother of 3 years old girl)
4. End use. End use is when mothers show and shares the stories with the public.

"I will promote my baby story in different kinds of channels for example I put the link of the page at status in Yahoo Messenger (YM) Instant Messenger or my email will include the signature of the link of the story too"( Mother of 3 years old girl)

"I will log-in to my YM in the morning (UK time) so my friends in Malaysia can ask me questions and I am able to answer it on the spot, this is suitable time because my baby would be still sleeping." (Mother of 3 years old girl)

"My friends like to response on the live chatter box in my blog. So I reply to their questions or sometimes real-time chat at the box too" (Mother of 1 year old boy)

\section{SOCIAL FACTORS BEHIND THE NARRATIVE MAKING PRACTICE}

Gubrium (2008) recommends to the narrative researcher to study the social relationships around the script [23]. Interestingly, we found lack of interaction and responses from participants' family in the comment section of the mediasharing space. Most of the comments there are from friends. Participants mention that family members like to comment over the phone or prefer face-to-face interaction. One way to study this phenomenon is by interviewing the participants about their family background and the context defining the mother-family relationship. This emphasis on revealing the social factors behind the narrative making practice is important because we argue that use of technology will be determined more by social motivations than by technological innovation. Our analysis of these aspects was guided by Bengtson's framework on intergenerational family relationship [24].

\section{Family Contact.}

All the participants of this research are the mother's of the baby; as a result, people most contacted are more on the maternal side. This close relationship adhere them to update their website.

"The relationship more closer to my mum compared to my dad. She is like a friend to me, I can tell her almost anything in terms of problems... when I have not updated my social pages she will SMS and ask, how I am, or everything is ok? So feel like responsible to update her about my family and especially about my baby, because she is the central of our communication." (Mother of 3 years old girl)

"If my mom is in Malaysia she is an active user because she want to see my baby's pictures, but if my mom is in the UK (visiting) she is not an active user but still looking at it anyway, she likes to see the pictures and do what she likes for example reading the comments people give to the post." (Mother of 3 years old girl)

2. Family Support. Some of the young parents did set up the computer at their parent's house with Internet facilities to overcome the cost of communication before migrating to the UK.

"Before we came here, my husband already set up broadband, $P C$ and webcam at my parents-in-law's house... beside for the purpose of video conferencing, they can also see sour latest news in the UK from our blog too, so it will save budget because developing photo and posting cost from the UK is expensive." (Mother of 3 years old girl)

3. Family Structure. Family structure reflected in number, type and geographic proximity of family member. We noticed that their mother (of the young mothers) are in the average age of 55 to 65 years old and in average have less than five 
grandchildren, so the relationship of grandparents interested to know about their grandchildren is higher.

"In terms of ranking, my baby if from my husband side, he is the $20^{\text {th }}$ grandchildren but for my side he is the second so my mother is much more interested to know about my baby. My mother-in-law she is quite old and besides she is not healthy too"(Mother of 1 year old girl)

4. Family Sharing Resources. Some grandparents who live alone in the rural area depending on the other siblings who has Internet access at the office to print out the picture for them.

"My mom has to wait for my sister who studies at university to go home if she wants to see the pictures because she has easy access to the Internet. My sister will develop the photo and show to my mother. So it takes times because in my hometown we do not have the Internet connection. The same goes with my parents-in-laws, they have to wait for my brother in-laws who is working to go back home during weekend to see the baby's picture."(Mother of 4 months baby and two boys)

5. Personal Incentives and Motivations. Some participants keep on continuously update their baby stories because it is their hobby either because they like to write story or like to take photo or video of her baby.

"I just like writing maybe; I have been writing a novel before but yet not published. Besides using written text I can express more. But not all I revealed in the public. I did tell everything to my mum using phone call"' (Mother of 3 years old girl).

According to participants, living in the UK with the gloomy weather most of the time would create depression. By creating the art of narrative could override the depression.

"When my husband went to school I feel so bored so I'm thinking of making something interesting and come across with baby narrative after looking at friend's Fotopages and keep on until now"(Mother of 4 months baby and two boys)

6. Spouse Support. In modern society it is more common that the wife has to work or study, and therefore the husband helps with child care. Four young mothers (two students and two professional) mentioned that the husband also help with baby story too by taking the video or photos or even write the story too.

"My husband records the video while I will do funny action to make the baby laugh" (Mother of 5 months baby and 4 years old boy)

"I would say that my husband is very helpful in maintaining the social space while I have no time to update it sometimes due to the work commitment (Mother of 3 years old girl)

\section{DISCUSSION}

We discuss here the results of our study and how they help to respond to our research question. The first research question on this study is 'What are the types of stories in baby storytelling?'. From the content analysis we found that young mothers tend to post more photos compared written text or record video. Photo is one of the favourites media because it is faster to share. Capturing portraits and outing are their favourite snaps while taking photos. Some of our participants mentioned that sometimes the family just want to go outing purposely for photo shooting session. Besides, this also would encourage family members to brainstorm other activities to enhance family gathering.

Our second research question is to investigate the narrative practices among young mother. The interview results show that the type of media depends on the task. If the mother would like to capture the baby talking or pronouncing words, video is the suitable channel to use. Most of the participants like to capture picture plus written text. Six out of nine participants combined the user of these two media. We believe that the lifecycle of the process of baby story will continue with the family support and the environment of the young mother.

Interestingly, in terms of the media choice, and in particular the selection of media-sharing type, we found that some participants just follow their friends while others compared and chose a certain media with the influence of the spouse.

"I did my own research, I compared Fotopages and Blogger, I would prefer blog because in blog we can write more." (Mother of 1 year old boy)

"We compared Friendster, Fotopages and Blogger. But my husband said Fotopages takes longer time to upload compared to Blogger. He doesn't like the appearance; he likes something like a webpage, so that's why he likes Blog" (Mother of 3 years old girl).

\section{CONCLUSION}

There are an increasing number of young mothers actively taking pictures of their babies and posting them on the Internet. This is said to have created the 'Photo Mama' phenomenon. Saiful Nang, co-founder of 'thePhotoMama.com' (launched on 1 January 2009) and claimed that 'thePhotoMama.com' is the first online community that will teach mothers about photography. Despite the explosion of baby story telling in social spaces and family communication in cyberspace, there are not many studies investigating media-based baby storytelling. We found several interesting phenomena from our study:

1. The type of media used depends on the types of story being attempted which follow the result of Media Richness Theory.

2. Majority of the young mothers prefers to post picture compared to written text or video. This is because amateur picture is easy to capture and requires less time to uploading to the social space.

3. The content that is shared through social space with extended families depends on the age of the babies. The older the baby becomes the more the content shared focuses on baby's physical activities.

4. There is a tendency to report only special events, which are perceived to be events that happen outside the homes. Routine home activities are only chosen as the last resort when there is nothing else to report.

From the analysis, we can propose some functions we believe new social space technology would need to support. Firstly, the social space can provide extra services which are related to the use of the content and the making of physical objects (e.g. photo albums, or selected photo to be in frame, photo on mug, poster or scrapbooks). Secondly, the social spaces can be more interactive and facilitate the contribution of multiple authors. This can encourage other family members to participate in the baby story making, even being able to put together the story by themselves based on content provided by the parents. Thirdly, privacy and access control mechanisms must be enhanced because some pictures are not suitable to view to the public. Finally, new systems can allow parents to use their creativity with combination of media (words, picture or video) to make the story more exciting.

Our ongoing study focuses now on the analysis of narratives using different types of media, the benefits for creators and the reactions from family members living away. We aim at developing a conceptual framework and design guide for rich- 
media forms of computer-mediated-communication (CMC) tools to create storytelling for family communication.

\section{ACKNOWLEDGMENTS}

We would like to thanks all the participants who volunteer participating in this study.

\section{REFERENCES}

[1] Stafford, L., Maintaining long-distance and crossresidential relationships-by Laura Stafford. Journal of communication, 2006. 56(3): p. 630.

[2] House, N.A.V., Flickr and public image-sharing: distant closeness and photo exhibition. CHI '07: CHI '07 extended abstracts on Human factors in computing systems, 2007.

[3] Nardi, B.A., et al., Why We Blog? Communications of the ACM, 2004. 47(12): p. 41-46.

[4] Corson, C., Grandparenting from a Distance. 2001: Oregon State University.

[5] Gonzalez, V. and S. Kurniawan, On new media for intergenerational communication: The case of Geriatric1927. (ISTAS 08), Fredericton, Canada 25-28 June 2008 IEEE Press, 2008.

[6] Harley, D. and G. Fitzpatrick, Creating a conversational context through video blogging: A case study of Geriatric 1927. . Journal of Computers in Human Behavior, 2008.

[7] Daft, R. and R. Lengel, Information richness: A new approach to managerial behavior and organization design. Research in Organizational Behavior, 6, 191-233., 1984.

[8] Madge, C. and H. O'Connor, Parenting gone wired: empowerment of new mothers on the internet? Social and Cultural Geography 7, 199-220, 2006.

[9] Ministry of Higher Education. Statistic Malaysian Student Abroad. 2008 [cited; Available from:

http://www.mohe.gov.my/web_statistik/statistik_pdf_2008 _05/msd_5-1.pdf.

[10] Abdullah, K., N.M. Noor, and S. Wok, The perceptions of women's roles and progress: A study of malay women. Social indicators research (2008):1-17., 2008.

[11] Department of Statistic Malaysia. Malaysia Population and Housing Census. 2000 [cited; Available from: http://www.statistics.gov.my/english/frameset_census.php
[12] Madden, M., Digital Footprints: Online identity management and search in the age of transparency. Pew Internet \& American Life Project, 2008.

[13] Sweeney, C. Twittering From the Cradle, New York Times. 2008 [cited; Available from: 11 Sept 2008.http://www.nytimes.com/2008/09/11/fashion/11Tots. $\mathrm{html}$.

[14] Brian, L.M. and M. Guzdial, iTell: supporting retrospective storytelling with digital photos, in Proceedings of the 6th conference on Designing Interactive systems, University Park, PA, USA. 2006.

[15] Bødker, S., et al., Co-operative design perspectives on 20 years with the Scandinavian IT design model. Proceedings of Nordi CHI 2000, Stockholm, 2000.

[16] Herring, S.C., Content analysis for new media: Rethinking the paradigm. In: New Research for New Media. Innovative Research Methodologies Symposium Minneapolis, University of Minnesota 2004: p. 47-66.

[17] Frohlich, D. and M. Jones, Audiophoto narratives for semi-literate communities. ACM interactions Nov-Dec 2008

[18] Khalid, H., Studying user experience in photo sharing. Volume 2 Proceedings of the 19th British HCI 2005, Napier University, Edinburgh, 5-9 September 2005., 2005.

[19] Cohen, K.R., What does the photoblog want? Media, Culture \& Society, 2005. 27(6): p. 883-901.

[20] Munce, C., Consumer Digital Imaging Survey, Photo Marketing Association. Photo Marketing Association International, 2000.

[21] Hyvärinen, M., Analysing Narratives and Storytelling in Social Research Methods, J. Brannen, P. Alasuutari, and L. Bickman, Editors. 2008, Thousand Oaks and London: Sage. p. 447-460.

[22] David, K., et al., Understanding videowork, in Proceedings of the SIGCHI conference on Human factors in computing systems. 2007, ACM: San Jose, California, USA.

[23] Gubrium, J.F. and J.A. Holstein, Narrative Ethnography. Handbook of Emergent Methods, ed. P.L. Sharlene Nagy Hesse-Biber. 2008: Guilford Press. 241-264.

[24] Bengtson, V.L., Intergenerational Solidarity in Aging Families: An Example of Formal Theory Construction. Journal of marriage and the family, 1991. 53(4): p. 8 